\title{
Structural stability predicts the binding mode of protein-ligand complexes
}

Maciej Majewski, $†$ and Xavier Barril*,†‡

† Institut de Biomedicina de la Universitat de Barcelona (IBUB) and Facultat de Farmacia, Universitat de Barcelona, Av. Joan XXIII 27-31, 08028 Barcelona, Spain

¥ Catalan Institution for Research and Advanced Studies (ICREA), Passeig Lluis Companys 23, 08010 Barcelona, Spain

*E-mail: xbarril@ub.edu 
Supplementary Information - Structural stability predicts the binding mode of protein-ligand complexes

Table S1. Classification of complexes from Iridium and SERAPhiC datasets.

\begin{tabular}{|c|c|c|}
\hline \multirow{2}{*}{ Category } & \multicolumn{2}{|c|}{ PDBid } \\
\hline & Iridium DS & SERAPhiC DS \\
\hline $\begin{array}{l}\text { Simulated } \\
\text { structures }\end{array}$ & 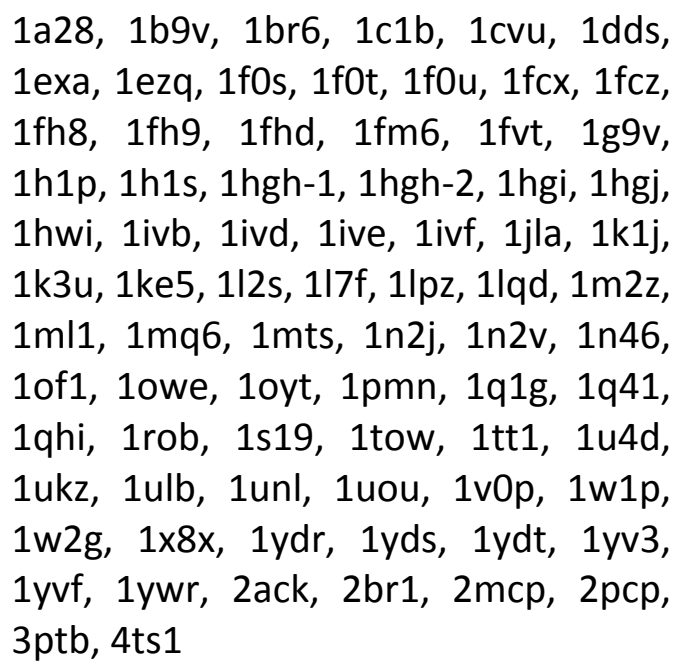 & $\begin{array}{l}\text { 1e2i_a, 1e2i_b, 1f5f, 1f8e, 1h46, } \\
\text { 1k0e, 1mlw, 1ofz_a, 1ofz_b, 1r5y, } \\
\text { 1sd1, 1tku, 1w1a, 1ynh, 2bkx, 2brt, } \\
\text { 2f6x, 2fgq, 2hdq_a, 2hdq_b, 2i5x, } \\
\text { 2iba, 2j5s, 2p1o, 2q6m, 2uy5, 3eko }\end{array}$ \\
\hline $\begin{array}{l}\text { Simulation } \\
\text { failure }\end{array}$ & 1ai5, 1gm8 & $1 \times f g$ \\
\hline $\begin{array}{l}\text { No hydrogen } \\
\text { bond }\end{array}$ & $1 \mathrm{ctr}, 1 \mathrm{fl} 3,1 \mathrm{p} 2 \mathrm{y}$ & $\begin{array}{l}\text { 1sqn, 1ui0, 1uwc, 2cix, 2qwx, 3c0z, } \\
3 d s x\end{array}$ \\
\hline Not drug like & 1fjs, $1 \mathrm{fm} 9,1 \mathrm{fq} 5,1 \mathrm{gwx}, 1 \mathrm{hgg}, 1 \mathrm{pso}$ & - \\
\hline Metal ion & 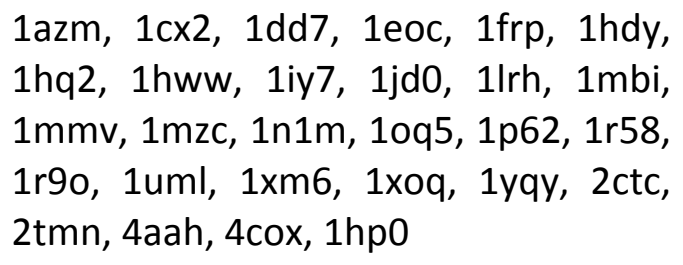 & $\begin{array}{l}1 \mathrm{~m} 2 \mathrm{x}, 1 \mathrm{~m} 3 \mathrm{u}, 1 \mathrm{~s} 5 \mathrm{n}, 1 \mathrm{t} 0 \mathrm{l}, 1 \mathrm{wog}, \\
1 \mathrm{x} 07,1 \mathrm{y} 2 \mathrm{k}, 1 \mathrm{yv} 5,2 \mathrm{aie}, 2 \mathrm{fdv}, 2 \mathrm{ff} 2, \\
2 \mathrm{gg} 7,2 \mathrm{gvv}, 2 \mathrm{rdr}, 2 \mathrm{v} 77,2 \mathrm{zvj}\end{array}$ \\
\hline $\begin{array}{l}\text { Additional } \\
\text { ligand }\end{array}$ & $1 \mathrm{~d} 3 \mathrm{~h}, 1 \mathrm{hnn}, 1 \mathrm{hvy}, 1 \mathrm{pbd}, 1 \mathrm{sq} 5$ & 1fsg, 1pwm, 1yki, 2b0m, 2bl9 \\
\hline
\end{tabular}


Supplementary Information - Structural stability predicts the binding mode of protein-ligand complexes

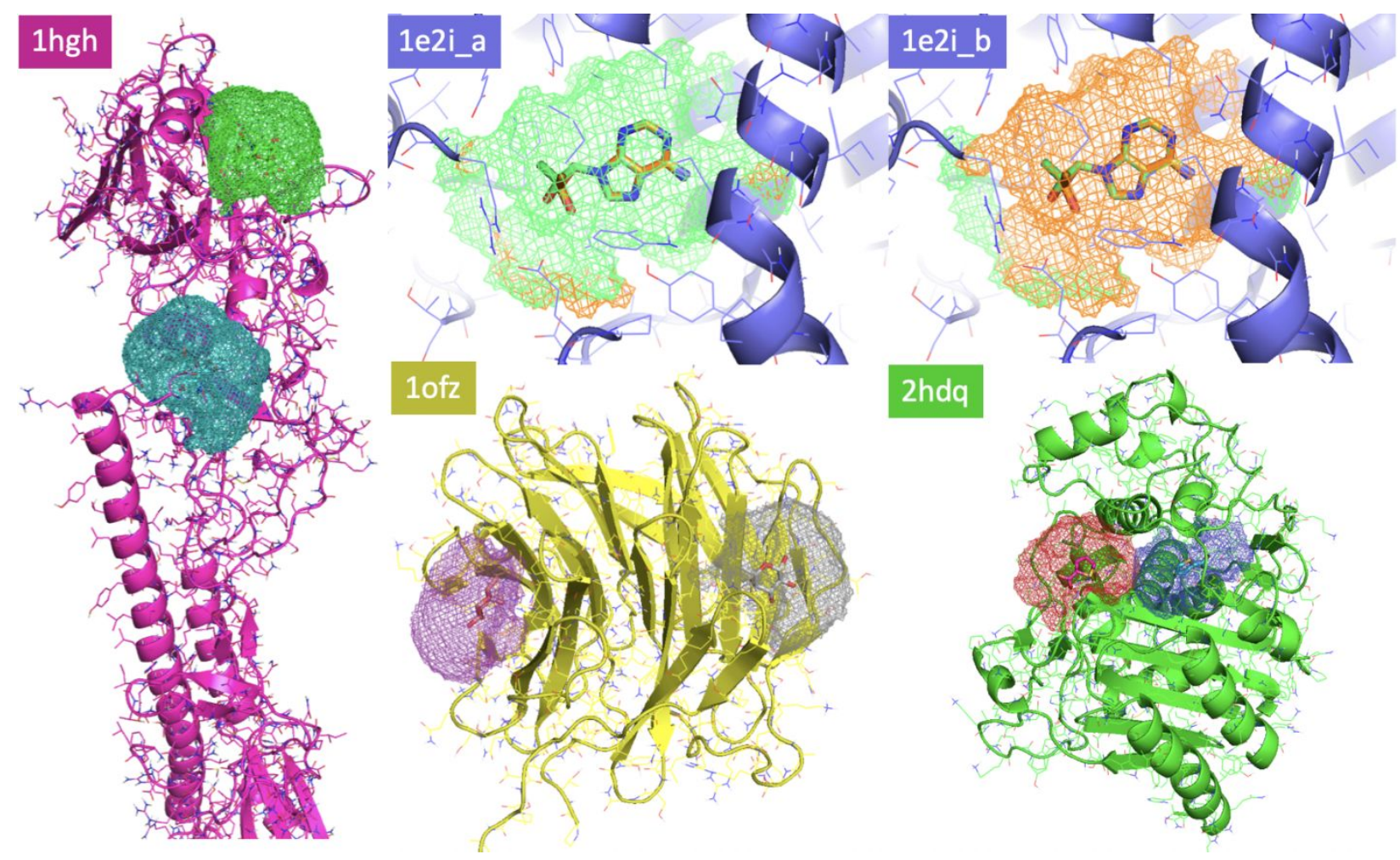

Figure S1. Binding cavities with generated grid of the complexes that possess two binding pockets

(1hgh, 1ofz, 2hdg) or with two enantiomers in the same binding pocket (1e2i).
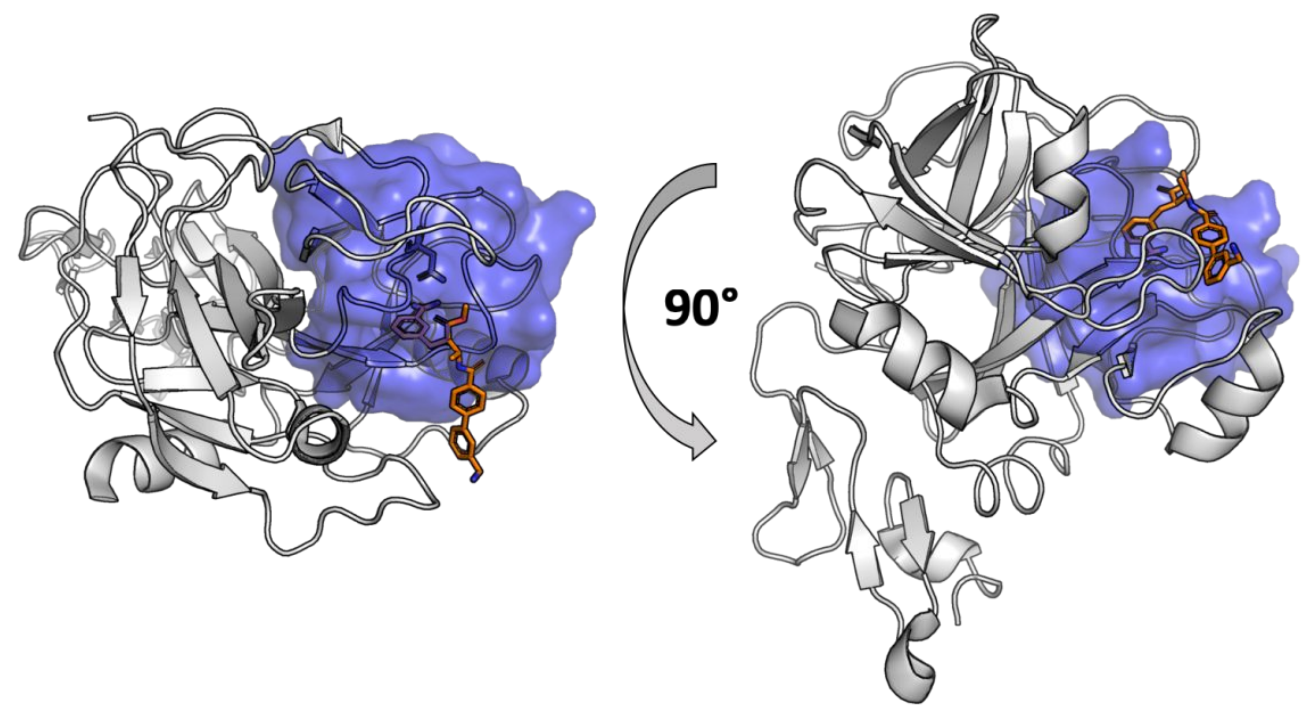

Figure S2. An example of chunk (blue surface) created for PDBid: 1eqz and based on the hydrogen bond (black dashed line) formed by ligand (orange sticks) and residue D188 (grey sticks). 
Supplementary Information - Structural stability predicts the binding mode of protein-ligand complexes

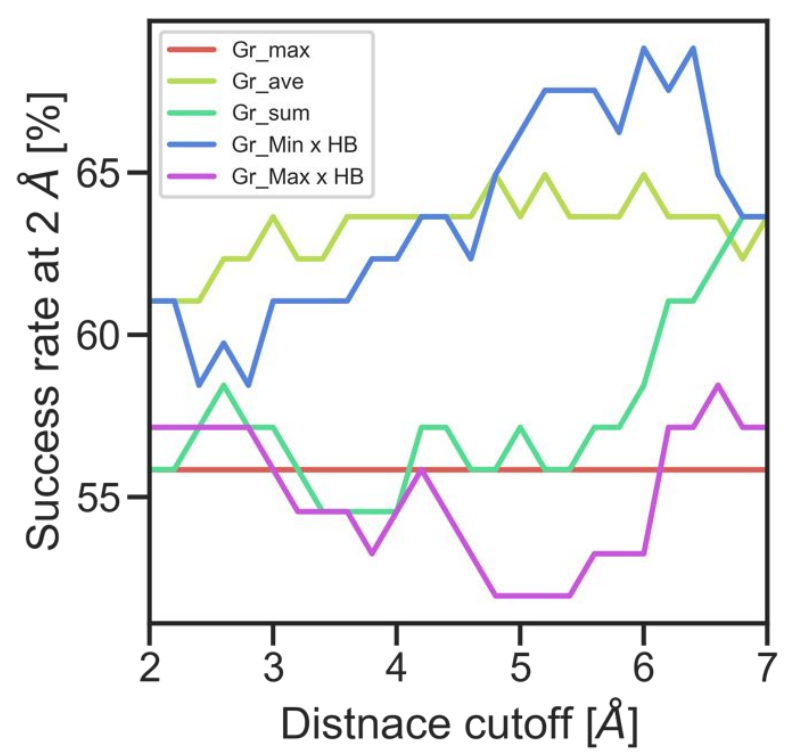

Figure S3. The plot representing success rate at $2 \AA$ in function of distance cutoff used for the group definition for Iridium dataset. The stability score of a group is selected based on definition presented in Methods, and the final score for the molecule is calculated as a maximum of group values. 
Supplementary Information - Structural stability predicts the binding mode of protein-ligand complexes
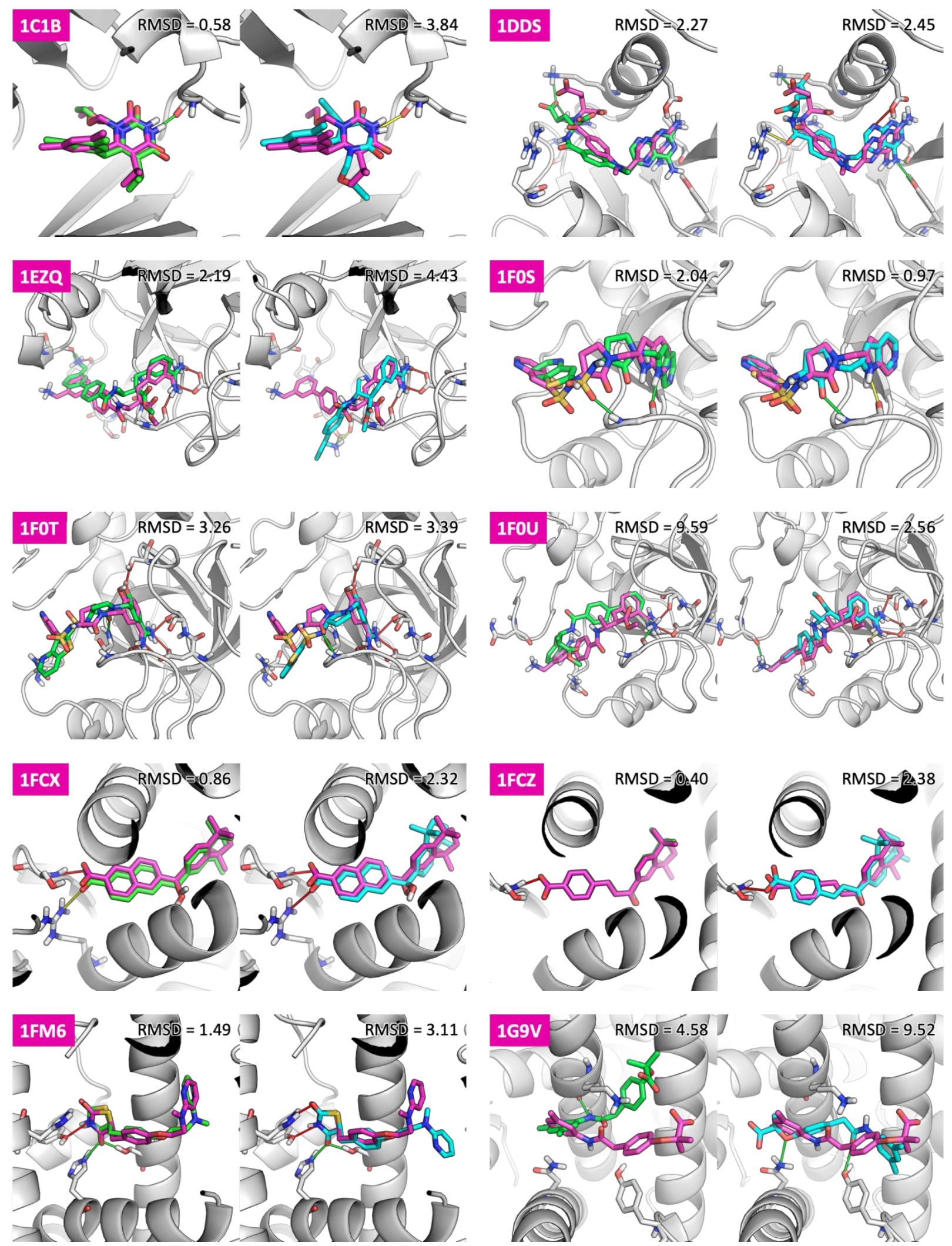

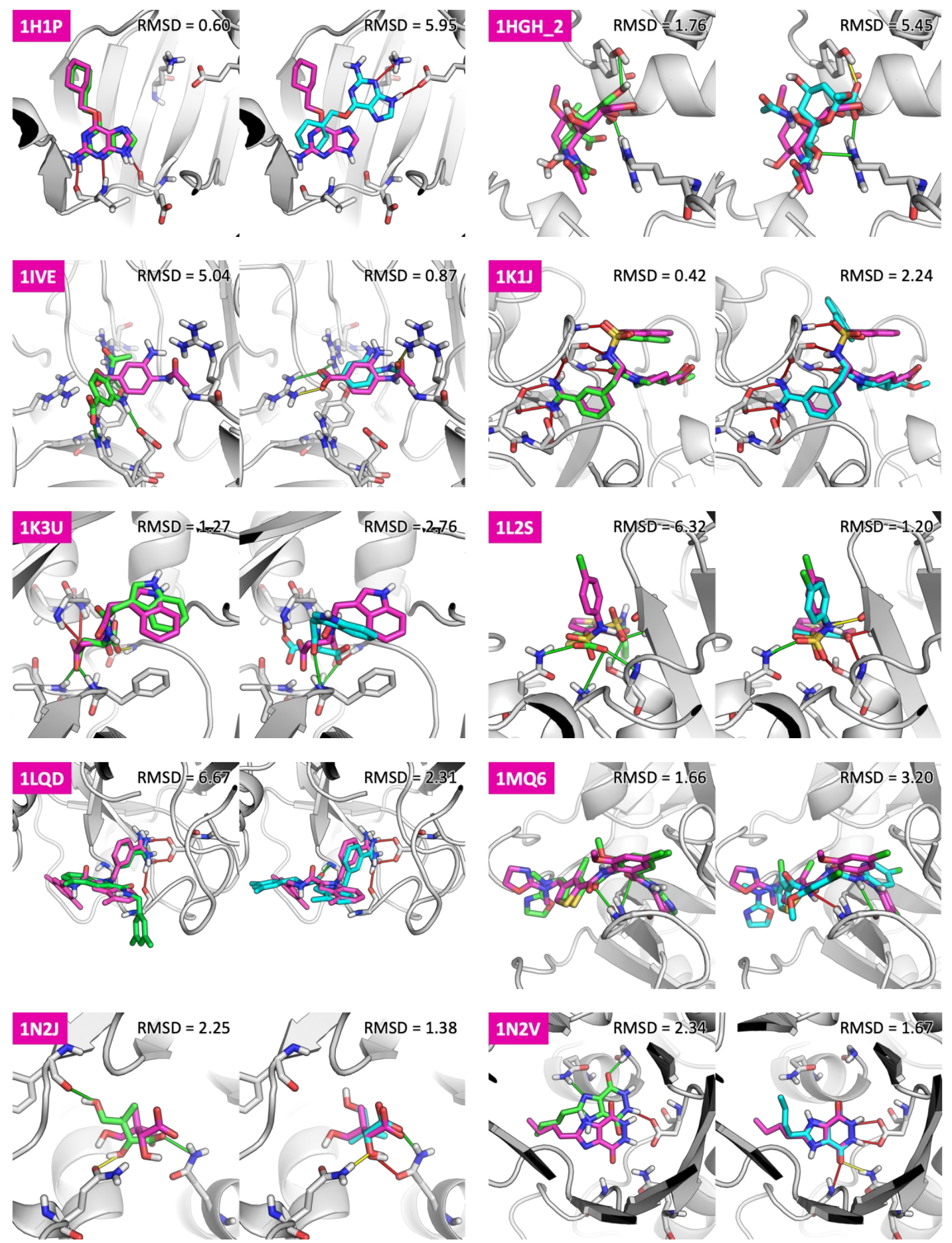
Supplementary Information - Structural stability predicts the binding mode of protein-ligand complexes
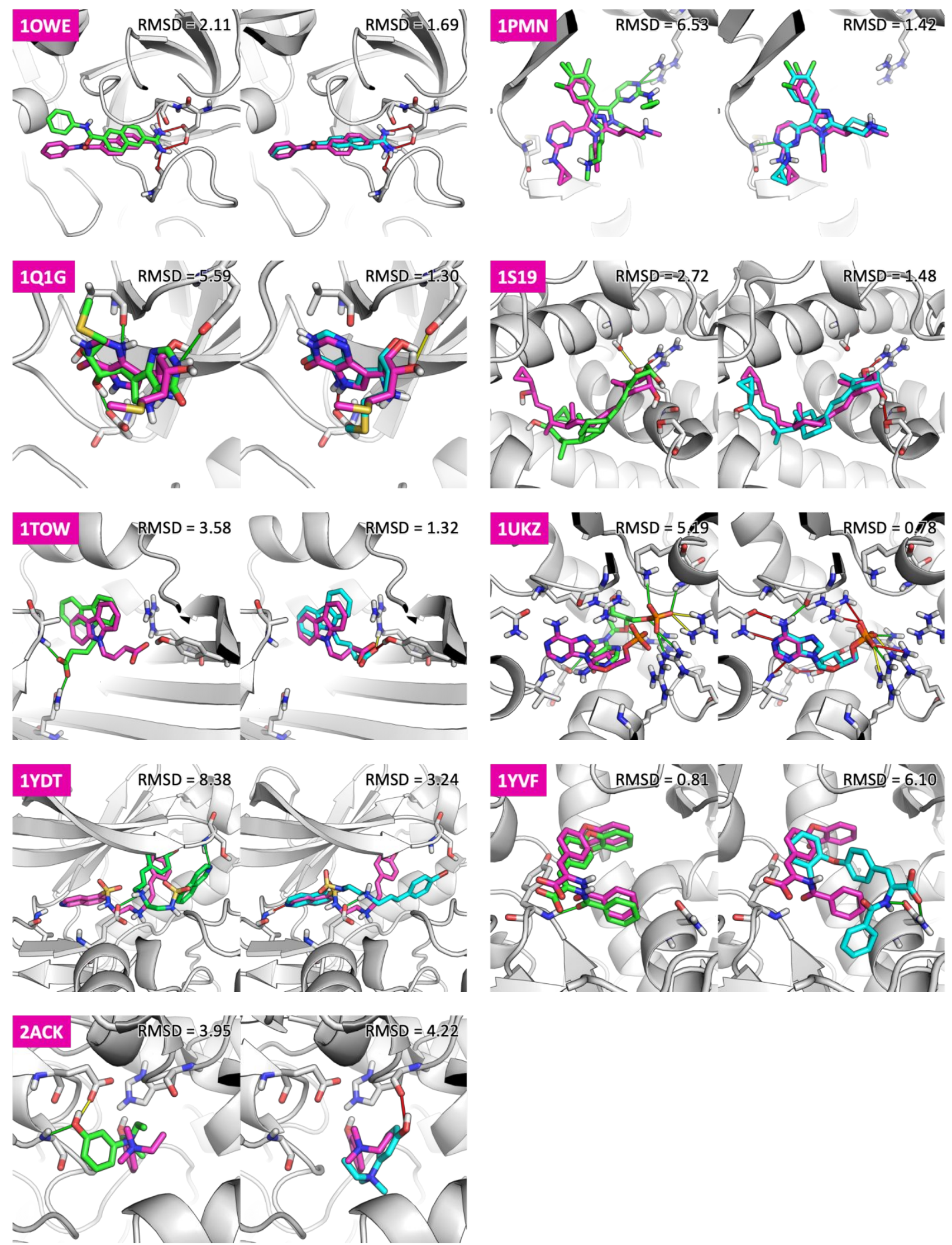

Figure S4. Predicted binding modes (BM) of ligands listed in Table 1. On the left side of each panel BM selected by rDock depicted in green, and on the right side BM selected by DUck depicted in cyan. True binding modes are depicted in magenta. 
Supplementary Information - Structural stability predicts the binding mode of protein-ligand complexes

\begin{tabular}{|c|c|c|c|c|}
\hline Sim. Time [ns] & 1.4 & $0.5 \times 2$ & 0.5 & $0.5 \times 2$ \\
\hline Wall clock time [min] & 48 & $19 \times 2$ & 15 & $19 \times 2$ \\
\hline & Equilibrate & $\mathrm{SMD}_{0}^{300 \mathrm{~K}}$ & & $\mathrm{MD}_{N} 300 \mathrm{~K}$ \\
\hline
\end{tabular}

Figure S5. Time performance of the simulation with a graph representing each step. "Wall clock time" was calculated for the average system of 25000 atoms (chunk, ligand and solvent, all including hydrogens) simulated on NVIDIA Tesla M2090 GPUs. For a single hydrogen bond, the complete simulation of such system takes 18.2 GPU hours. Depending on the size of the system, the simulation time can vary by about $20 \%$. A pose makes on the average 4 hydrogen bonds, and we simulated 5 poses per ligand. The total estimate of the time necessary to simulate 1 ligand is 364 GPU hours (72.8 $\mathrm{h}$ per pose). The computational performance can be further optimized by changing simulation length, number of replicas, type of enhanced sampling method, number of particles, integration time-step using atomic mass repartitioning, better parallelization, next generation GPUs, etc.

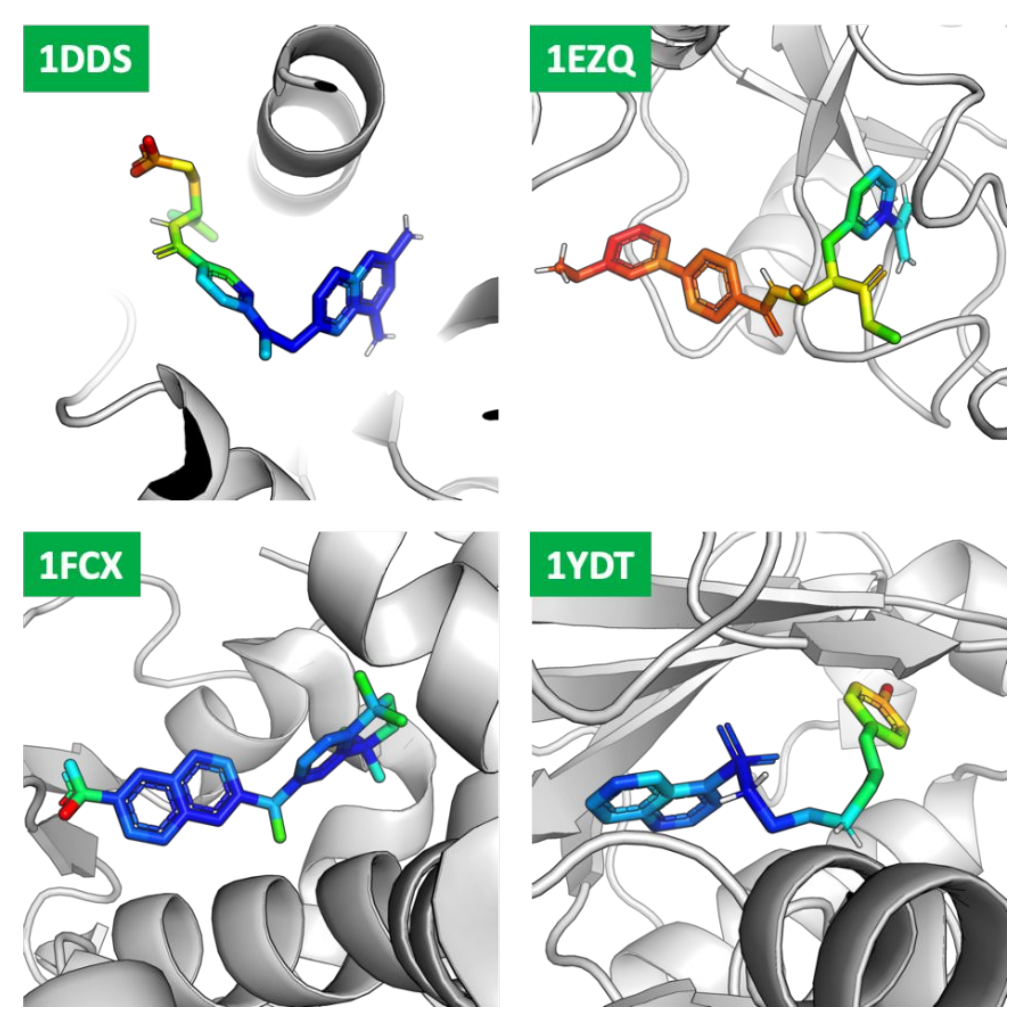

Figure S6. Crystal structures of complexes depicted in Figure 5. The ligands have been coloured based on their relative temperature factors, blue indicating colder (ordered) and red hotter (flexible) regions. 
Supplementary Information - Structural stability predicts the binding mode of protein-ligand complexes

Table S2. Error estimation of success rates extracted from Figures 2 and 3. The errors were calculated using bootstrapping. The success rate of each scoring variant was calculated 10000 times for a random sample with replacement (size of sample 26 for SERAPhiC and 77 for Iridium). Then error was calculated as a standard deviation across all samples.

\begin{tabular}{|c|c|c|c|}
\hline & \multicolumn{2}{|c|}{ SERAPhiC } & Iridium \\
\hline Scoring method & SR $(2 \AA)$ & SR $(1.5 \AA)$ & SR $(2 \AA)$ \\
\hline rDock & $42.3 \pm 9.7$ & $38.5 \pm 9.5$ & $75.3 \pm 4.9$ \\
\hline DUck_max & $57.7 \pm 9.7$ & $50.0 \pm 9.8$ & $55.8 \pm 5.6$ \\
\hline DUck_min & $46.2 \pm 9.8$ & $30.8 \pm 9.1$ & - \\
\hline DUck_ave & $69.2 \pm 9.1$ & $46.2 \pm 9.8$ & $66.2 \pm 5.4$ \\
\hline DUck_sum & $69.2 \pm 9.1$ & $50.0 \pm 9.8$ & $66.2 \pm 5.4$ \\
\hline DUck_group & - & - & $76.6 \pm 4.8$ \\
\hline random & $21.7 \pm 7.8$ & $14.0 \pm 6.4$ & $23.6 \pm 4.7$ \\
\hline
\end{tabular}

